\title{
Method for Measuring Ultra-Short Distances Using RADARs for Burden Profile Measurement at Blast Furnace
}

\section{Chitresh Kundu ${ }^{1}$, Prabal Patra ${ }^{2}$, Bipan Tudu ${ }^{3}$, Dibyayan Patra ${ }^{4}$}

${ }^{1}$ TATA Steel, Instrumentation \& Control, Jamshedpur, Jharkhand, India ${ }^{2}$ TATA Steel, Instrumentation \& Control, Jamshedpur, Jharkhand, India.

${ }^{3}$ Jadavpur University, Kolkata, West Bengal, India.

${ }^{4}$ Vellore Institute of Technology, Vellore, Tamil Nadu, India.

* email: chitresh.kundu@tatasteel.com

\section{DOI: https://doi.org/10.30609/jeti.v4i2.12184}

\begin{abstract}
The operation of the furnace depends largely on the handling of gas distribution which in-turn is reliant on the burden surface profile. Measurement of burden profile has been a challenge because of the harsh conditions inside the furnace. Phased array radar will suffice this purpose with no moving parts; however, it imposes its own challenges. One of them is measurement of extremely short distances as the stockline in the blast furnace is just few meters away from the topmost part. Unlike conventional radar where the target is kilometre away the short distance measurement involves with time measured in nano seconds with pico-seconds resolution to achieve the desired accuracy. This paper implements a method for ultra-short distance measurement using radars for blast furnace application in steel plant.
\end{abstract}

Keywords: Blast Furnace, Profile, RADAR, Burden Distribution, Time Interval

\section{Introduction}

Blast furnace in steelmaking process is at present the main procedure for processing the iron ore in a steel plant. In more than 100 years of steel making, Blast Furnaces everywhere throughout the world are as yet considered as covert process [1] because of lack of instrumentation due to harsh conditions. Blast Furnace being the mother plant for a coordinated steel plant, any anomality will influence the overall production. Comparing all process variables that impact the furnace operation, burden surface profile is the most significant one to increase productive efficiency and reducing power resource consumption. In absence of any continuous reliable measurements for burden distribution profile, there exists a reliance on numerical models and approximations to operate the furnace [2]. Passive Radar technology that makes use of the signal reflection from a remote object is in use for 
about 100 years. The emitted radar signal can be sent out from a $360^{\circ}$ (omnidirectional) antenna in all directions or, highly focused, in one direction. For accurate distance measurement, it is mandatory to have a focused beam pointing at the target. The beam opening angle is typically in the range of 2 to 5 degrees. A smaller opening angle can only be achieved with very large antennas, not suitable to for the given size of distance measurement units in industrial process automation.

Obtaining a surface profile for blast furnace at an elevated accuracy, resolution and high data throughput is always a demanding task in the research field of metrology. Many non-contact methods have been proposed for surface profile measurement prior to this [3]. The laser and microwave are two other forms of electromagnetic waves that are commonly used for time-of- flight measurement, and which differ only by their wavelengths.

In the electromagnetic spectrum, the lower frequencies tend to have more penetration. Hence for this application where the environment is dusty the microwave frequencies will be ideal as the hot blast stirs up lot of powdery particles upwards.

Distances will be determined by measuring the time-of-flight of the radar signal from the emitter to the target and back to the receiver. The signals travel at the speed of light. For a $100 \mathrm{~m}$ stretch, the signal travels about $333 \mathrm{~ns}$. Accurate and reliable distance measurement in industrial environment beyond a certain range requires that the effect of objects ranging into the radar signal is eliminated.

The application of most measuring technologies has been impeded by the unforgiving conditions in blast furnaces. To accomplish accurate estimations, a few strategies have been endeavoured in the previous decades.

A method for high precision local positioning radar using an Ultra-Wide Band concept has been demonstrated [4]. The concept is based on standard Frequency modulated continuous wave radar principle combined with short pulses to fulfil the emission limits as per regulatory requirements. High accuracy in dense multipath environments is claimed using this concept for 1D, 2D and 3D localization. Frequency of operation used for demonstration is $7.5 \mathrm{GHz}$, with a bandwidth of $1 \mathrm{GHz}$ (satisfying the FCC criterion for being termed UWB). Common FMCW approach was thus combined with UWB techniques to make use of advantages of both ideas.

Distance measurement is using RTOF measurement after high synchronization. FMCW concept is utilized for system design, and a working model is demonstrated. In multipath situation, precise distance and velocity measurements were studied by [5] [6] using an FMCW approach. 
Performance synopsis of Circular geolocation using TOA approach and Hyperbolic geolocation using TDOA approach were presented by [7][8]. Detailed comparative analyses of four primary schemes used are discussed -

- Circular (TOA approach) - with or without knowing receiver cock

- Hyperbolic (TDOA approach) - At same /different time instants

Detailed study of the expected position error is also provided. Statistical channel models inferred from field trials are used to determine realistic error probabilities. Comparison is established in two different ways - strict \& average. In strict type, methods are investigated for a particular configuration of base stations w.r.t some mobile position, which in turn determines a given noise profile affecting Time of Arrival / Time Difference of arrival estimates. In average type, methods are evaluated in terms of the expected covariance matrix of the position error over an ensemble of random geometries, so that the comparison obtained finally is geometry independent. An Accurate UWB localization system using time difference of arrival approach is discussed in [9]. Designed at $8 \mathrm{GHz}$, the system practices a time difference of arrival method System is in compliance with FCC UWB regulations (Related to PSD and Bandwidth). It discusses and provides a clear comparison between FMCW and TDOA approaches. It also accounts for multipath and the measured accuracy is sub-cm, with potential for sub $\mathrm{mm}$ accuracy discussed. The utilized time domain measurements suppress multipath signals and can provide accuracies up to sub $\mathrm{mm}$ range. It can also be extended to $2 \mathrm{D}$ and $3 \mathrm{D}$ also.

Using time-of-flight based method is more accurate against the frequency domain measurements in low bandwidth applications. Particularly in burden profiling it can provide centimetre accuracy by measuring time intervals with picosecond resolution.

Accuracy in distance measurement is directly correlated to time difference measurement accuracy in time domain. The time of flight is measured in a radar system by calculating the difference between the transmitted (START) and received signal (STOP). In case of pulsed radar these signals are the transmitted pulse and the received echo. There are various techniques and methods for achieving precise and accurate time measurement with specific reference to pulse radar level measurement like the charging and discharging of capacitor [10] using the pulse train from the transmitted and received signal is used to measure the time interval. By provision the charging is kept faster than discharge. Though the method has the capability of attaining high resolution but in this attainment, there is a requirement of prolonged conversion time. Usually, the errors are outcomes of nonlinearity's and quantization. Averaging them may improve the output result, however 
additional delays is the factor to consider. There is another method where the capacitors voltage is held and performing the Analog to Digital conversion on this value [11]. Resulting voltage is directly proportional to the elapse time i.e. difference between the start and stop signal. This method is similar to a pulse stretching technique, with the difference that instead of the counter the ADC directly reads out the value. The measurement time can be reduced by speeding up the capacitor discharge. Time Stretching is a method involving two pulse trains [12]. The first pulse train consists of the transmitted signal with some Pulse Repetition Interval (PRI). With a marginally higher PRI, the second pulse train is a reference/sampling signal. Each point of the transmitted signal is multiplied point by point with the reference signal resulting in an intermediate frequency. The closer the difference between the signals, prolonged time expansion will be generated. ACAM Mess Electronic Gmbh [13] has introduced integrated circuits that can measure time intervals with very high resolution and accuracy. It uses the tapped delay lines having same Propagation delay $(\tau)$. Between the START and STOP signal, the state of the delay line is used for time interval measurement. This method is hight scalable and practical due to its low power and manufacturing cost

\section{Material and Methods}

With a typical blast furnace geometry as shown in Figure 1, using the concepts of radar, pulses are transmitted from the antenna, and they travel at the speed of light. Accuracy in distance measurement is directly correlated to time difference measurement accuracy in time domain.

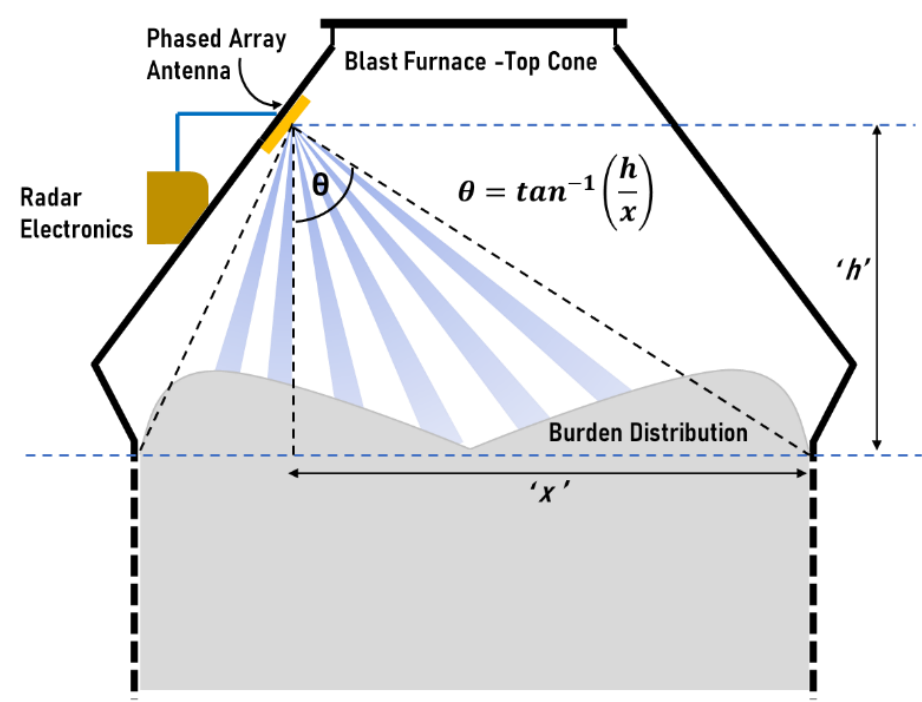

Figure 1 Blast furnace geometry

Typically, in the blast furnace scenario the maximum height for measurement ' $h$ ' is equal to the $5 \mathrm{~m}$ from stockline. A meter distance corresponds to return time of flight of $6.67 \mathrm{~ns}$. 
Hence for measuring $5 \mathrm{~m}$ the maximum time interval to be measured is $33.35 \mathrm{~ns}$. A one nanosecond pulse extends in space for $30 \mathrm{~cm}$. So, for accuracies desired in industrial measurements (of the order of $\mathrm{mm} / \mathrm{cm}$ ), the time interval measurement should also be very precise. Time interval measurement is the measurement of elapsed time between some designated Start and Stop phenomenon.

The important parameters to consider in designing the time interval measurement and for time interval meters are:

1. Minimum interval - The minimum time between consecutive pulses.

2. Minimum dead-time - The minimum time between the stop pulse and the next start pulse.

3. Minimum pulse width - The shortest pulse the circuitry will recognize

4. Measurement range - The longest possible time the instrument can measure

5. Readout speed - How fast the instrument can produce a result

In case of pulsed radar these signals are the transmitted pulse and the received echo. The signal of the emitting unit is received at the receiver. Any distance determination requires the exact measurement of the travel time of the signal.

In this paper, the Time to digital Convertion (TDC) chips has been used which is able to measure the short time intervals we expect in the radar level measurement instrument. ACAM Messelectronic has been researching time interval measurement in the picosecond range and some of the chips available to them is shown in Table 1

Table1: Types of time-to-digital converter chips available

\begin{tabular}{ccc}
\hline Model & Resolution & Range \\
\hline TDC-GP1 & $125 \mathrm{ps}$ & $2 \mathrm{~ns}-7.6 \mu \mathrm{s}$ \\
TDC-GP2 & $50 \mathrm{ps}$ & $3.5 \mathrm{~ns}-1.8 \mu \mathrm{s}$ \\
TDC-GPX & $81 \mathrm{ps}$ (I-mode) & Endless \\
& $41 \mathrm{ps}$ (G-mode) & $0 \mathrm{~ns}->10 \mu \mathrm{s}$ \\
& $27 \mathrm{ps}$ (R-mode) & $0 \mathrm{~ns}->10 \mu \mathrm{s}$ \\
& $10 \mathrm{ps}$ (M-mode) & $0 \mathrm{~ns}->10 \mu \mathrm{s}$ \\
\hline
\end{tabular}

The systems were designed and fabricated with the RF front end. For each of the section, The RF part and the Signal processing part are separately arranged as two different layers as shown in Figures $2 \& 3$ 


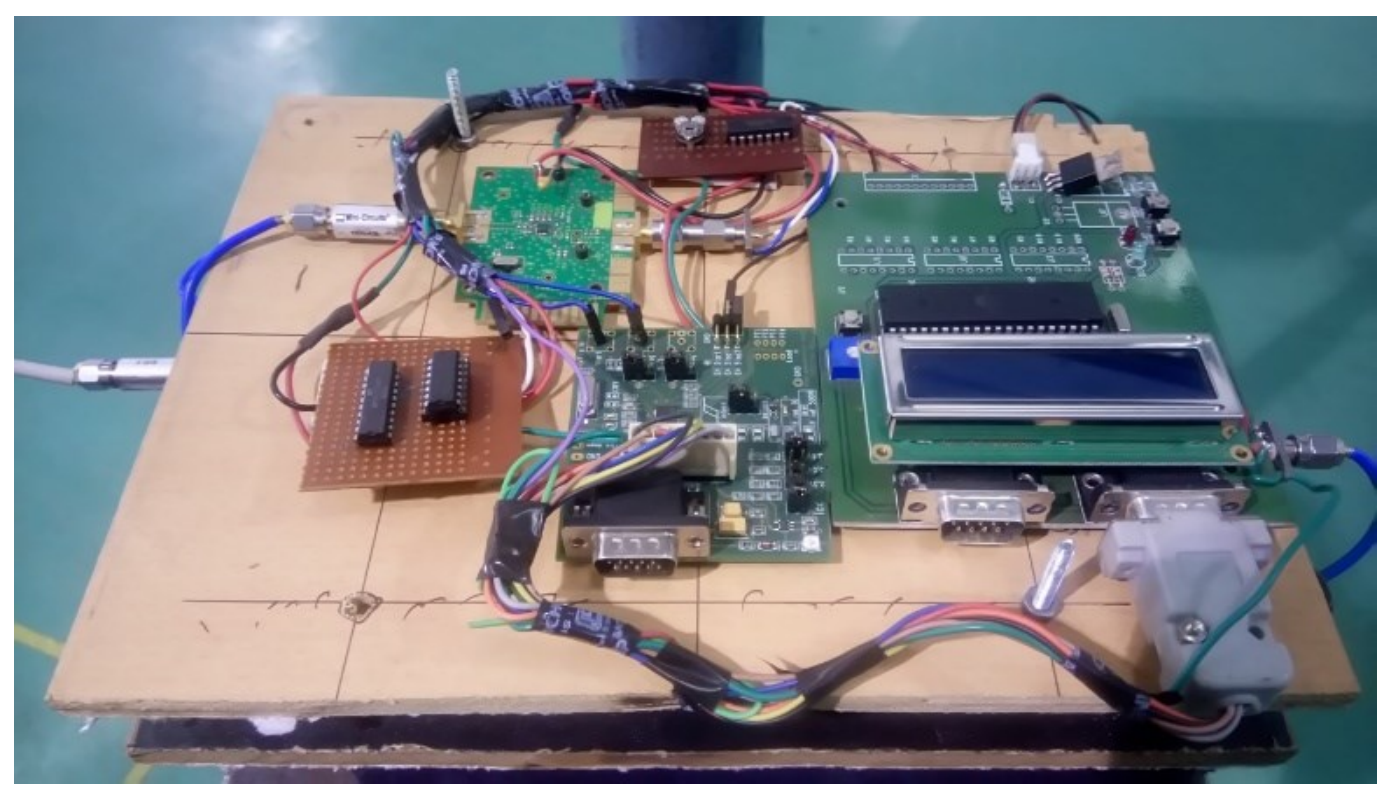

Figure 2 Signal processing Section

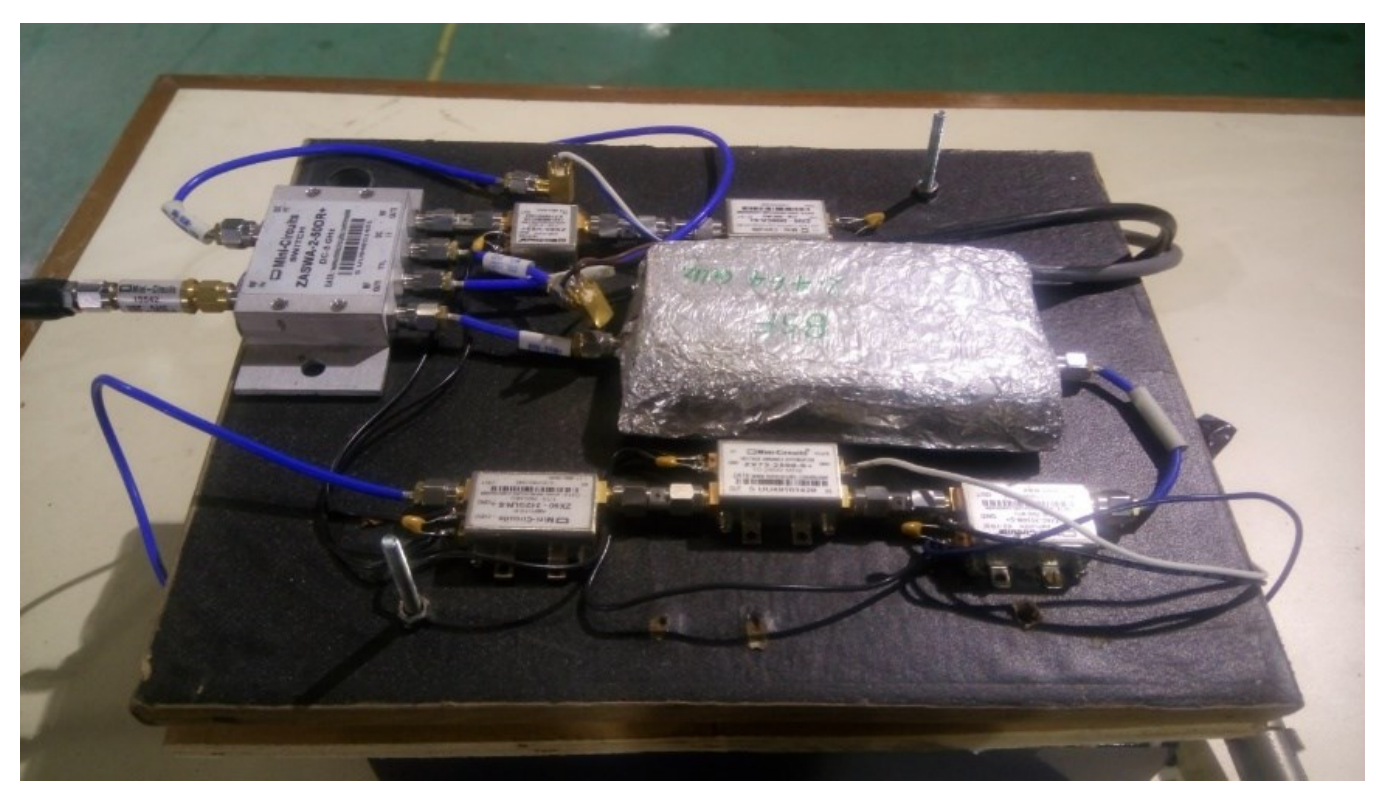

Figure 3 RF section of Transceiver

The pulses transmitting from the level measurement implementation travel at the speed of light as they are electromagnetic in nature. As the target medium is placed at a short distance of $2.5 \mathrm{~m}$, the travel time of pulses is less. Between the transmitted and received signal, the time interval (TI) is very short; therefore, precise TI measurement is essential for the pulse radar system.

The required accuracy of the system should be $1 \%$ of the complete scalable distance which contributes to an accuracy of $50 \mathrm{~mm}$. Along with it, there is requirement of aa very 
high resolution of $50 \mathrm{~mm}$, which in time domain parallels to a resolution of about $333 \mathrm{ps}$. Hence, the need to measure T1 very precisely to 333 ps.

Measurement between START and STOP occurrence of the elapsed time is the TI measurement. In pulsed radar scenario, the transmitted signal is pulse and the one received is echo. Detection of these transmitted pulses are thru detectors. Inputs of START and STOP can be either single or separate. The timing of the input pulses arises only when it crosses a definite threshold. The measured output is then converted into a digital value. This conversion is achieved by time-interval meter, time-to-digital converter or time counter as shown in Figure 4. T1 measurement between single transmitted and echo pulse is described as the one-shot measurement. Resolution of analogue or the Least Significant Bit (LSB) of digital limits the resolution of the one-shot measurement. But this resolution can be amended by averaging the $\mathrm{TI}$ in analogue and interpolating in digital. Averaging the T1 are based on factors that are random and as these random factors tend to zero, the resolution escalates to high values. Interpolation implicates calculation of estimated data between the discrete time data. The objective of the end result is enhanced resolution restricted by the noise.

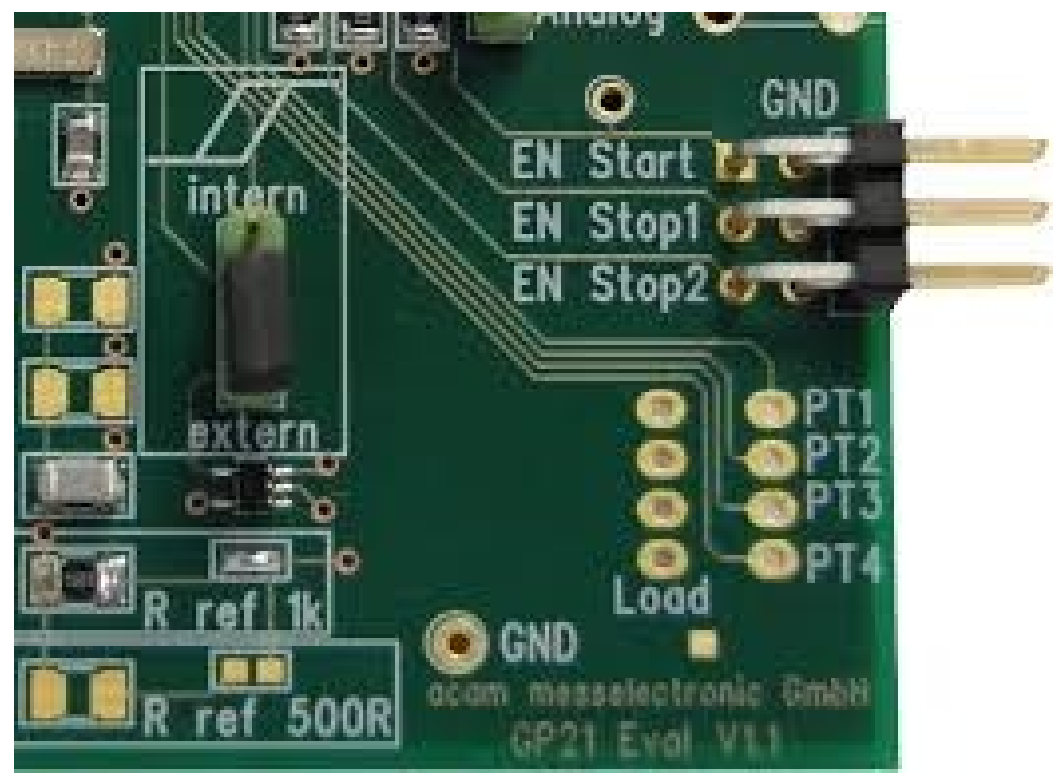

Figure 4 Time conversion circuit

\section{Results and discussion}

The objective is to obtain the accuracy and repeatability of the time-of-flight measurements. To obtain data for time-of-flight measurements a test setup has been developed by installing the system in parallel to a laser distance meter (LDM). The laser system is used to accurately measure the distance between the locations. As shown in Figure 5 , the setup consists of the Radar system (test equipment) with a corner reflector at opposite 
(fixed location). The other location can be moved thus varying the distance between them. The reading from the laser system i.e., the distance vales were compared against the timeof-flight measurements from the radar system.

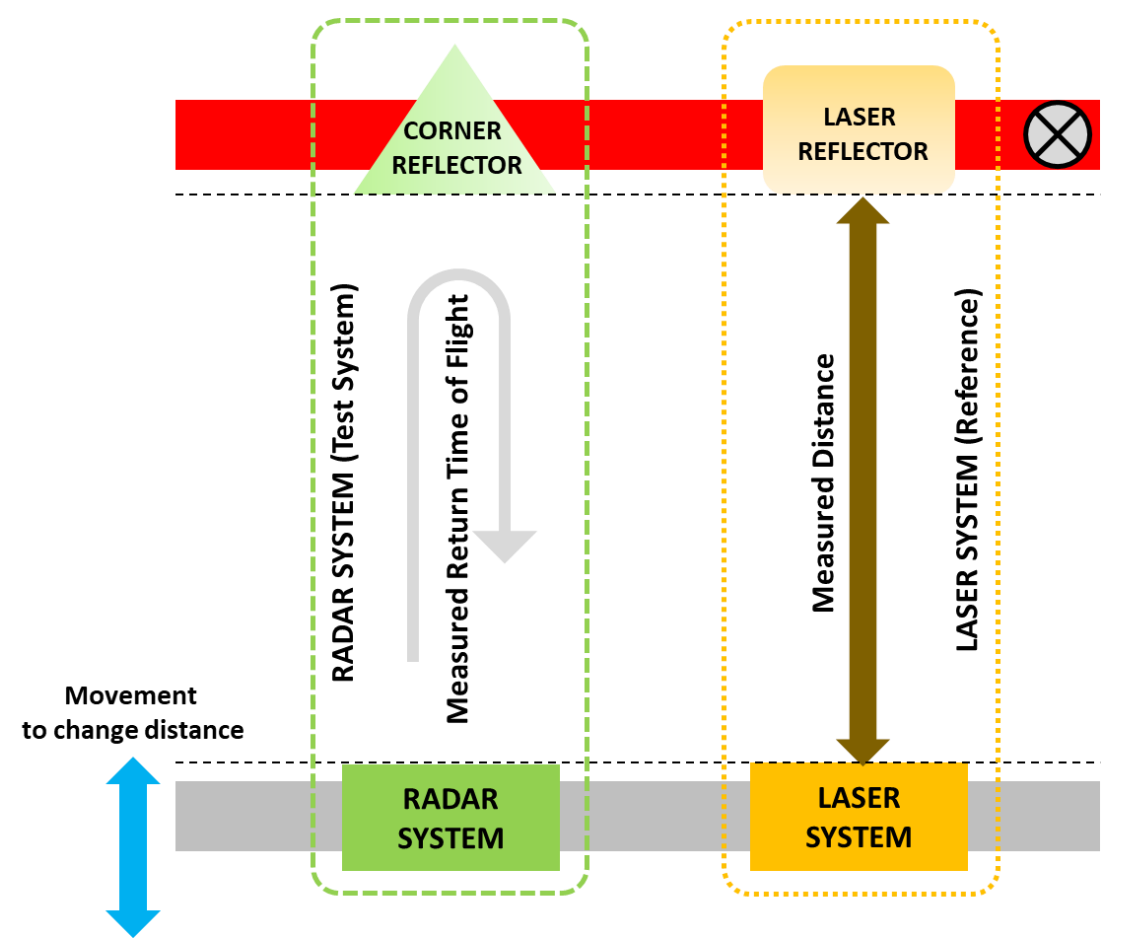

Figure 5 Block Diagram of Radar System Test Setup

Firstly, the station was kept static and by using LDM, the distance is measured as $3000 \mathrm{~mm}$. Multiple (10000 samples) time of flight readings were measured for the waves to travel between the antenna and backscatter plate. Figure 6 depicts the histogram of RTOF (return time of flight) of the readings thus taken. The histogram illustrates that the measurements follow a Gaussian distribution as expected.

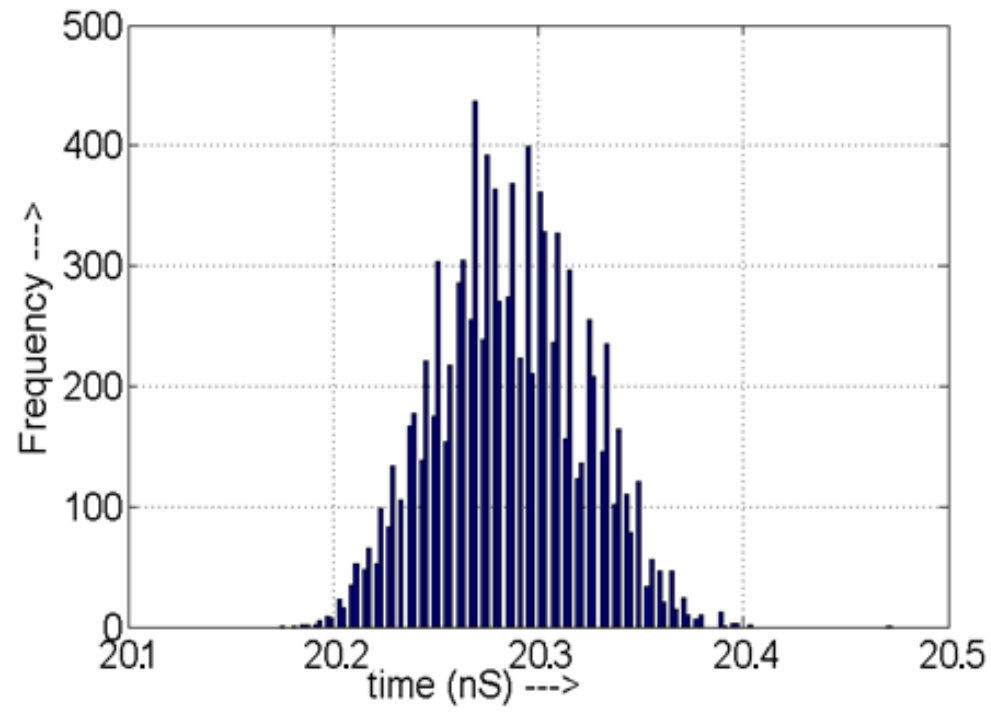

Figure 6 Histogram plot of the measured time intervals. 


\section{Normal and double resolution data comparison}

Further the time measurement circuitry was capable to measure the time intervals at much higher resolution. To obtain the results, the double resolution mode of the TDC was invoked. In the double resolution mode, the radar's resolution is lower than the normal mode. The readings are obtained for same static measurement conditions both in normal and double resolution modes as described in Table 2.

Table 2: Observations (Comparison of normal and double resolution data)

\begin{tabular}{ccc}
\hline Measurement Mode & Mean & Standard Deviation \\
\hline Normal & $20.27 \mathrm{~ns}$ & $122 \mathrm{ps}$ \\
Double Resolution & $20.25 \mathrm{~ns}$ & $55 \mathrm{ps}$ \\
\hline
\end{tabular}

Observations derived from Table 2 depicts that there is minimum deviation in the mean value whereas then standard deviation has significantly reduced.

In the next step of measurement, the tests using two closely separated known distances were conducted. For the readings, the distances were changed and it is measured as $3050 \mathrm{~mm}$ and $3100 \mathrm{~mm}$ respectively. These distances were chosen in accordance to the practical blast furnace scenario where the burden is in between $2800 \mathrm{~mm}$ to $3500 \mathrm{~mm}$ from the radar system. The time of flight corresponding to that has been shown in figure $7 \mathrm{a} \& 7 \mathrm{~b}$.

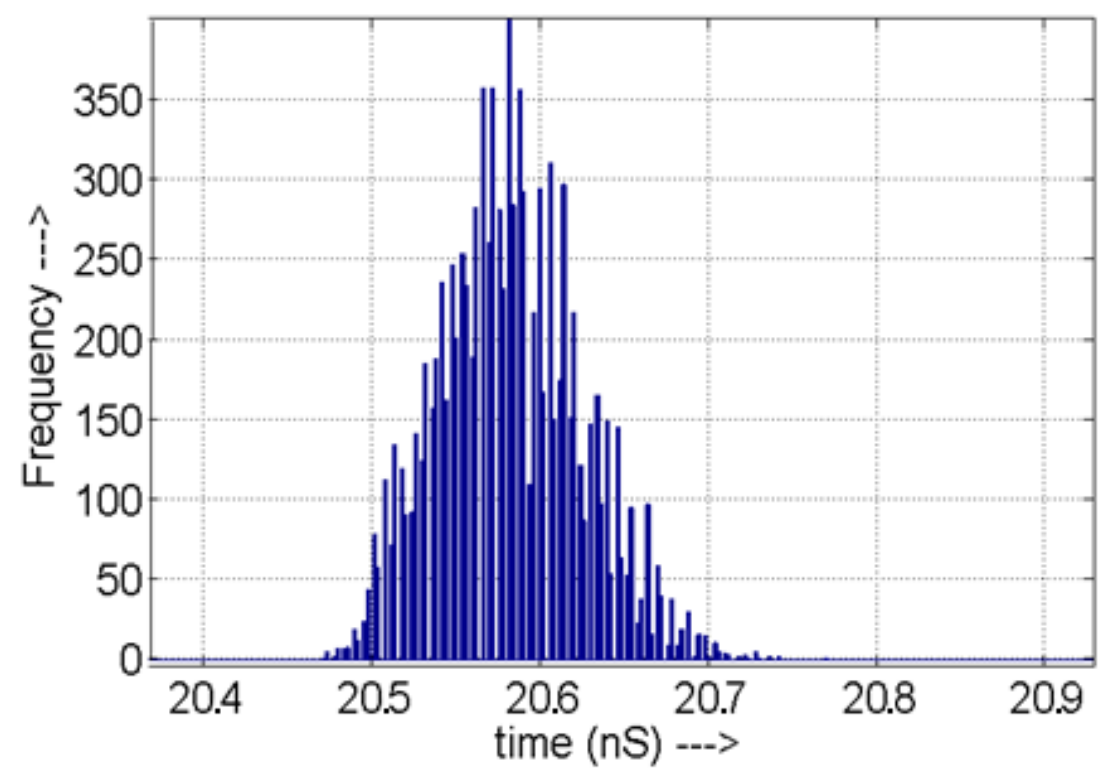

(a) 


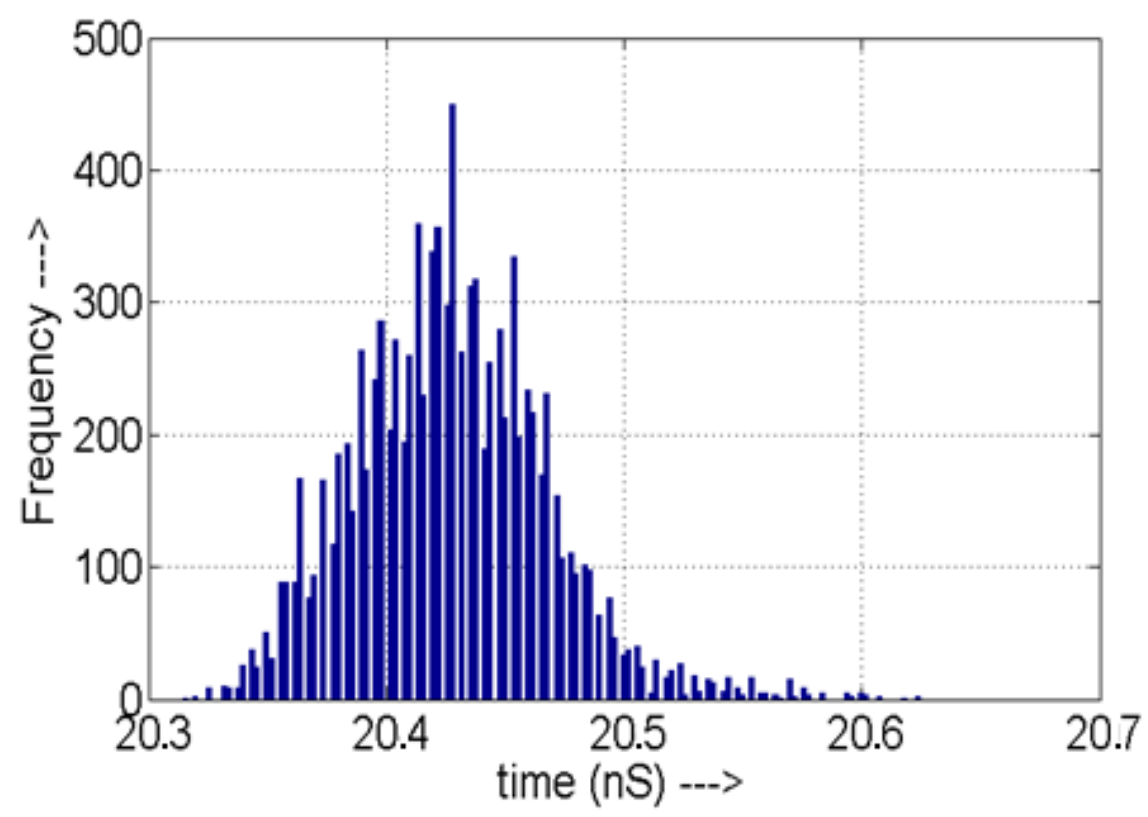

(b)

Figure 7: (a) Distance: $3100 \mathrm{~mm}$; (b) $3050 \mathrm{~mm}$

Further the measured values were compared with the theoretical values and shown in table 3. As the radar waves are microwaves the theorical time of flight has been calculated using the speed of light. Thus, any time-of-flight measurement can also be calculated back to the distance using the same formula. The error obtained in the measurement were less than $0.5 \%$ making the system extremely accurate for short distance measurement.

Table 3: Summary of measurement results

\begin{tabular}{ccc}
\hline Distance(mm) & 3050 & 3100 \\
Calculated time of flight (ns) & 20.334 & 20.668 \\
Measured time of flight & 20.431 & 20.580 \\
(ns) & & \\
Error (\%) & 0.477 & 0.425 \\
\hline
\end{tabular}

\section{Conclusion}

The proposed strategy provides utilization of radar in a typical blast furnace scenario where distance to be estimated for burden profiling is very short. It utilizes a successful radar framework for exact estimation of distance-based on return time of flight measurements. The method can be utilized for obtaining the data of the burden profile in steel plant. It is seen 
that the estimation is extremely accurate. The utilizations of the radars also make it a suitable technology for blast furnaces as it has very high dust and smoke penetration.

\section{Acknowledgment}

This paper and the research behind it would not have been possible without the exceptional support of the members at ICT Lab, Automation Division, TATA Steel, India. My colleagues and my superiors have looked over my transcriptions and answered with unfailing patience numerous questions.. I am also grateful for the insightful comments and. the generosity and expertise of one and all that have improved this study in innumerable ways and saved me from many errors.

\section{Bibliography}

[1] Masaaki Naito, Kanji Takeda, Yoshiyuki Matsui, Ironmaking Technology for the Last 100 Years: Deployment to Advanced Technologies from Introduction of Technological Know-how, and Evolution to Next-generation Process

[2] Park, Jong-In \& Jung, Hun-Je \& Jo, Min-Kyu \& Oh, Han-Sang \& Han, Jeong-Whan. (2011). Mathematical modeling of the burden distribution in the blast furnace shaft. Metals and Materials International. 17. 485-496. 10.1007/s12540-011-0629-7.

[3] M. Hattori, B. Iino, A. Shi,omura, H. Tsukiji and T. Ariyama, "Development of Burden Distribution Simulation Model for Bell-less Top in a Large Blast Furnace and Its Application,” ISIJ International, vol. 33, No. 10, 1993.

[4] B. Waldmann, R. Weigel, and P. Gulden, "Method for high precision local positioning radar using an ultra wideband technique," in IEEE MTT-S Int. Microw. Symp. Dig., Atlanta, GA, Jun. 2008, pp. 117-120.

[5] S. Roehr, P. Gulden, and M. Vossiek, "Method for high precision clock synchronization in wireless systems with application to radio navigation," in IEEE Radio Wireless Symp., Long Beach, CA, Jan. 2007, pp. 551-554.

[6] M. Kossel, H. R. Benedickter, R. Peter, and W. Bachtold, "Microwave backscatter modulation systems," in IEEE MTT-S Int. Microw. Symp. Dig., Boston, MA, Jun. 2000, pp. $1427-1430$.

[7] S. Roehr, P. Gulden, and M. Vossiek, "Novel secondary radar for precise distance and velocity measurement in multipath environments," in Eur. Radar Conf., Munich, Germany, Oct. 2007, pp. 182-185.

[8] M. Vossiek and P. Gulden, "Switched injection locked oscillator: A novel versatile concept for wireless transponder and localization systems," IEEE Trans. Microw. Theory 
Tech., vol. 56, no. 4, pp. 859-866, Apr. 2008.

[9] C. W. Yak, Z. Lei, S. Chattong, and T. T. Tjhung, "Timing synchronization and frequency offset estimation for ultra-wideband (UWB) multiband OFDM systems," in IEEE Int. Pers. Indoor Mobile Radio Commun. Symp., Berlin, Germany, Sept. 2005, pp. 471-475. [10] R. Zetik, J. Sachs, and R. Thomae, "UWB localization-Active and passive approach [ultra wideband radar]," in IEEE Instrum. Meas. Technol. Conf., Como, Italy, May 2004, pp. 1005-1009.

[11] M. Vossiek, L. Wiebking, P. Gulden, J. Wieghardt, C. Hoffmann, and P. Heide, “Wireless local positioning," IEEE Micro, vol. 4, no. 4, pp. 77-86, Dec. 2003.

[12] Peter Devine. "Radar Level Measurement - The Users guide". Vega Controls Ltd., 2000.

[13] K Maatta and $\mathbf{J}$ Kostamovaara. "High-precision time-to-digital converter for pulse time-of-fight laser radar applications", IEEE Transactions on Instrumentation and Measurement, 47:521 536, 1998. 